\title{
Treatment of Limbal Melanocytoma with Full Excision and a Fresh Homologous Corneoscleral Graft in a Dog
}

\author{
João Antonio Tadeu Pigatto', Luciane de Albuquerque', Anita Marchionatti Pigatto ${ }^{2}$, Alessandra Fernandez da Silva', \\ Marcela Torikachvili', Eduarda Valim Borges de Vargas' \& David Driemeier ${ }^{1}$
}

\begin{abstract}
Background: Limbal melanoma has been diagnosed in dogs and due to progression may cause vision loss and eyeball removal. Definitive diagnosis is made through histopathological examination. Therapeutic options include full thickness resection and repair by homologous corneal tissue, synthetic graft material, and enucleation. In this report, we describe a case of limbal melanocitoma in a dog that has been treated successfully with fresh homologous corneoscleral graft.

Case: A 5-year-old female Labrador was referred to the Ophthalmology Veterinary Section of the Federal University of Rio Grande do Sul (UFRGS), Porto Alegre, Brazil, with a history of a pigmented mass located on the left eye. Ophthalmic examination revealed a pigmented mass located at the left temporal limbus with corneal involvement. Surgical excision followed by reconstruction using fresh homologous corneoscleral was recommended. The patient was premedicated with acepromazine $(0.05 \mathrm{mg} / \mathrm{kg}, \mathrm{IM})$ and meperidine $(20 \mathrm{mg} / \mathrm{kg}$, IM). Anaesthesia was induced with propofol (10 mg/kg, IV) and maintained with isoflurane. Atracurium $(0.2 \mathrm{mg} / \mathrm{kg}$, IV) was administered to maintain a central eye position. The mass and a free margin were removed by full-thickness corneoscleral resection. A corneoscleral graft was harvested from a dog that had been euthanised for reasons unrelated to this study and sutured with 9-0 polyglactin 910 using a simple interrupted pattern. The mass was immediately fixed in $10 \%$ neutral buffered formalin and submitted for histological sectioning and routine staining. Based on the histopathological analysis it was confirmed limbal melanocytoma. Postoperative treatment consisted of topical administration of $0.3 \%$ flurbiprofen every $6 \mathrm{~h}$ for 15 days, and a combination of topical ciprofloxacin/ dexamethasone eye drops every $6 \mathrm{~h}$ for 30 days. Systemic carprofen $(4 \mathrm{mg} / \mathrm{kg}$ per day, VO) was prescribed for 10 days. Topical tropicamide was used twice daily for 1 week. Cyclosporin $0.2 \%$ eye drops were applied twice daily for 2 months. Examination of the left eye two months after surgery revealed decreased corneal vascularization, and the results of pupillary light response and vision testing were normal. The values of intraocular pressure remained normal in all postoperative evaluations. The patient was followed for 36 months postoperatively, during which time there was no recurrence.

Discussion: Limbal melanomas are the most common ocular melanomas in dogs. Most of them develop slowly and are located closely to the superior limbus. The tumors tend to grow more rapidly in younger dogs and more slowly in older dogs. Labrador Retrievers and German shepherds appear to be affected more frequently than other breeds. The present case involved a 5-year-old Labrador breed. The clinical presentation included a pigmented limbal mass extending into the adjacent cornea, sclera and conjunctival tissue. A tissue biopsy is necessary to confirm the diagnosis. In the present case, the diagnosis of limbal melanocytoma was based on clinical signs and confirmed by histopathological examination. The choice of treatment is influenced by the tumour size and location, availability of equipment and materials, clinician expertise and the cost of treatment. Some techniques for removing the mass and repairing the resultant corneoscleral defect has been described. In the present case, due to the location and size of the mass and the absence of intraocular invasion, surgical removal including a margin of normal tissue was performed. With this surgical procedure, the intention was to preserve the eyeball and maintain vision. In this case, surgical excision of a limbal melanocytoma combined with homologous corneoscleral graft was effective for repairing a full-thickness corneoscleral defect and preserving ocular function.
\end{abstract}

Keywords: canine, fresh corneoscleral graft, melanocytic neoplasia. 


\section{INTRODUCTION}

Limbal melanocytomas are benign, heavily pigmented masses originating from corneoscleral melanocytes [12]. These tumours are predominantly reported in dogs, cats and horses [4,7]. The clinical signs are typically minimal, with the mass often found incidentally, although local corneal invasion, epiphora and mild conjunctival irritation may occur [12]. Treatment is required to prevent intraocular spread, which ultimately results in loss of vision in the eye [9]. Previously reported treatments for dogs with limbal neoplasia include surgical resection and laser photocoagulation, as well as a combination of surgical treatment, laser photocoagulation and cryotherapy $[1,2,10]$. Deep surgical excision accompanied by a tissue graft early in the course of growth offers the best prognosis. Eye wall defects following fullthickness mass removal in dogs have been reported to be repaired using frozen corneoscleral, synthetic and porcine small intestinal grafts $[5,6,9,11]$. Penetrating scleroplasty with fresh corneoscleral grafting has been reported for the treatment of anterior uveal tumours in humans [8]. However, fresh corneoscleral grafts have not yet been used to repair canine cornea and sclera after excision of limbal neoplasia. This report describes the successful use of a fresh homologous corneoscleral graft to repair a corneoscleral defect resulting from the excision of a limbal melanocytoma in a dog.

\section{CASE}

A 5-year-old female Labrador dog was referred to the Ophthalmology Veterinary Section of the Federal University of Rio Grande do Sul (UFRGS), Porto Alegre, Brazil, for evaluation of a pigmented mass on the left eye. The dog's owner reported that the mass had increased in the previous 6 months. Ophthalmic examination revealed a pigmented mass located at the left temporal limbus (Figure 1). Schirmer tear test I values were $20 \mathrm{~mm} / \mathrm{min}$ in the right eye and $18 \mathrm{~mm} /$ min in the left eye. The intraocular pressure, estimated with a rebound tonometer, was $19 \mathrm{mmHg}$ bilaterally. Menace, dazzle and pupillary reflex tests indicated normal vision in both eyes. There were no other ocular clinical signs associated with the pigmented lesion. Ultrasonography and gonioscopy revealed no intraocular invasion of the tumour. The right eye was normal, and all laboratory exams were also normal.
Surgery was recommended to remove the mass and reconstruct the globe. For $48 \mathrm{~h}$ prior to surgery, the left eye was treated topically with $0.03 \%$ flurbiprofen (Ocufen $0.03 \%)^{1}$ ophthalmic drops and ciprofloxacin (Ciloxan $0.3 \%)^{2}$ ophthalmic drops administered every $6 \mathrm{~h}$. Surgery was performed under general anaesthesia using an operating microscope. The patient was premedicated with acepromazine (Apromazin 0.2\%) ${ }^{3}$ $[0.05 \mathrm{mg} / \mathrm{kg} \mathrm{IM}]$ and meperidine (Dolantina) ${ }^{4}$ [20 mg/ $\mathrm{kg} \mathrm{IM]}$. Anaesthesia was induced with propofol (Diprivan $)^{5}[10 \mathrm{mg} / \mathrm{kg}]$ and maintained with isoflurane (Isoflurano) $^{6}$. Atracurium (Tracur) ${ }^{7}[0.2 \mathrm{mg} / \mathrm{kg} \mathrm{IV}]$ was administered to maintain a central eye position. The dog was positioned in a dorsal position, and the affected eye was routinely aseptically prepared. A lateral canthotomy was performed to better expose and approach the mass. A lid speculum was inserted and the globe was stabilised with a fixation ring. The initial $3.2-\mathrm{mm}$ incision was made in the clear cornea at the 11 o'clock position close to the limbus, and $0.5 \mathrm{~mL}$ of adrenaline $(1: 1000)$ was injected into the eye. A viscoelastic substance was used to maintain the anterior chamber and protect the corneal endothelium. The conjunctiva and Tenon's fascia were dissected. The mass and a 3-mm free margin were removed by full-thickness corneoscleral resection using a corneal trephine and corneal scissors. A corneoscleral graft was harvested from a dog that had been euthanised for reasons unrelated to this study. Immediately after euthanasia, with the aid of an 8-mm corneal trephine and conjunctival scissors, a scleral corneal button was removed. A homologous corneoscleral graft similar in size to the corneoscleral defect was trimmed and sutured with 9-0 polyglactin 910 using a simple interrupted pattern. The retracted conjunctival flap was repositioned and sutured over the limbus with simple interrupted sutures of 9-0 polyglactin 910 . The viscoelastic substance was removed from the anterior chamber using a Simcoe irrigating/ aspirating cannula. The canthotomy was closed using 5-0 monofilament nylon. An Elizabethan collar was placed on the patient to minimize self-trauma. The resected mass was fixed in $10 \%$ buffered formalin and submitted for histological examination in the Sector of Veterinary Pathology (SVP), Faculdade de Veterinária (FaVet), Universidade Federal do Rio Grande do Sul (UFRGS), Porto Alegre, RS, Brazil. Postoperative treatment consisted of topical administration of $0.3 \%$ flurbiprofen every $6 \mathrm{~h}$ for 15 days, and a combination 
of topical ciprofloxacin/dexamethasone eye drops every $6 \mathrm{~h}$ for 30 days. Systemic carprofen (Rimadyl) ${ }^{8}$ [4 mg/ $\mathrm{kg}$ per day VO] was prescribed for 10 days. Topical tropicamide (Mydriacyl solução) ${ }^{9}$ was used twice daily for 1 week. Cyclosporin (Ciclosporina Pomada 0.2\%) ${ }^{10}$ $0.2 \%$ eye drops were applied twice daily for 2 months.

One day after surgery, the dog was comfortable and the menace response remained intact. Minimal inflammation was observed, the anterior chamber depth was normal, and the dog showed no signs of discomfort. By 7 days postoperatively, mild corneal oedema was observed, and superficial corneal vascularization was noted adjacent to the graft. One month after surgery, corneal vascularization extended to the medial edge of the graft, and granulation tissue elevated the temporal margin of the graft. At 6 weeks, the dog seemed comfortable and the amount of granulation tissue had decreased. Examination of the left eye 2 months after surgery revealed decreased corneal vascularization, and the results of pupillary light response and vision testing were normal. The values of intraocular pressure remained normal in all postoperative evaluations.

Histopathologic examination revealed the presence of tumour cells across the full thickness of the cornea and sclera. Histologically, they showed deep, heavily pigmented cells, which formed a cohesive mass. The histopathologic diagnosis was limbal melanocytoma. The dog was subjected to regular ophthalmic examinations four times a year, and visually presented a transparent cornea and no evidence of neoplasm recurrence after 3 years of follow-up (Figure 2).

\section{DISCUSSION}

Melanocytic limbal neoplasms in dogs have been reported $[1,4,12]$. A bimodal age distribution

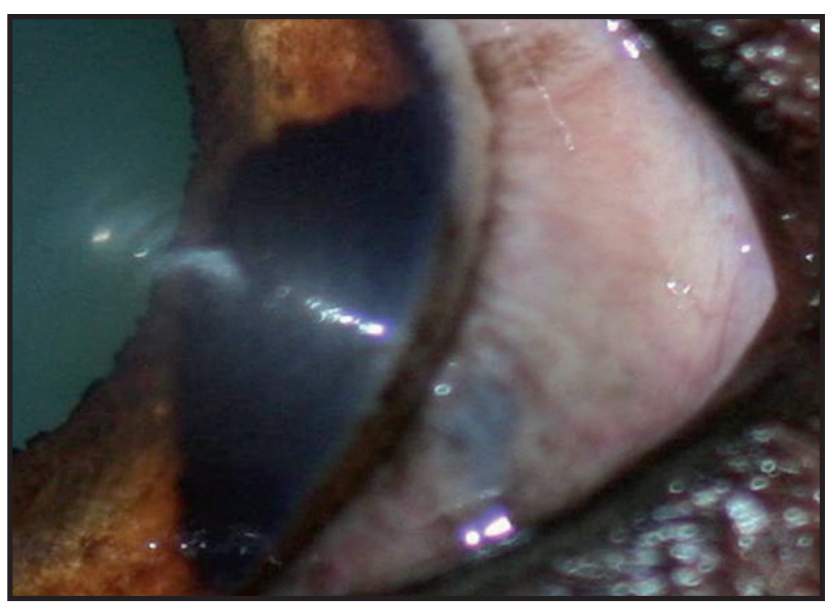

Figure 1. Appearance of the left eye exhibiting the smooth mass at the temporal limbus. Note that the pigmentation extends into the cornea and sclera adjacent. with distinct peaks between 3-4 and 7-10 years of age has been reported in dogs presenting melanocytic ocular tumours [6]. Breed predisposition for German Shepherds and Golden and Labrador Retrievers has been described $[3,6]$. The present case involved a 5 -year-old Labrador breed. The clinical presentation included a pigmented limbal mass extending into the adjacent cornea, sclera and conjunctival tissue. Previous reports have suggested a predilection for the dorsolateral quadrant of the eye [1]. Possible differential diagnoses for limbal melanocytoma in dogs include limbal melanoma, epibulbar melanocytoma, conjunctival melanoma and uveal melanoma with extrascleral extension [6]. In the present case, the diagnosis was based on patient's history and clinical signs and was confirmed by histopathological examination of the mass after surgical excision. Diagnosis of limbal melanocytoma is made by excisional biopsy $[9,11]$. This case is similar to other previously reported cases of canine limbal melanocytoma in terms of the clinical features and histopathological characteristics.

Treatment of limbal neoplasia includes lamellar or full-thickness excision of the mass with safety margins, followed by replacement with grafting $[2,5,6,9,11]$. However, enucleation is recommended in cases where the tumour shows intraocular extension. Enucleation can be curative, but is only recommended in eyes in which the melanoma has invaded the anterior chamber, filtration angle or uveal tract, resulting in painful intraocular disease [1]. In this case, as shown in the ultrasound examination and gonioscopy, there was no intraocular tumour invasion, therefore, excision of the mass with a safety margin followed by a fresh homologous corneoscleral graft was proposed. There are few clinical cases reported in the literature regard-

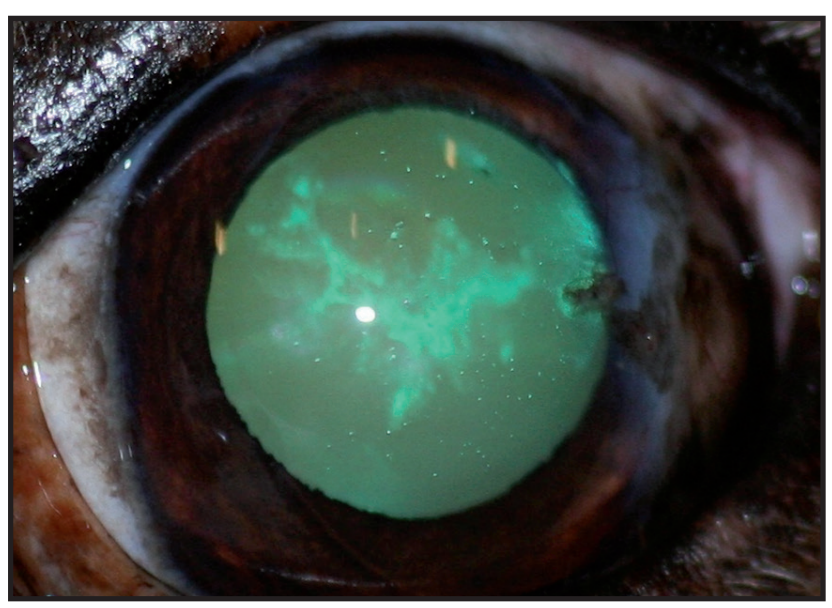

Figure 2. Appearence of the left eye three years after surgery. Note the good corneal transparency. 
ing the successful treatment of dogs with melanocytic neoplasms $[2,5,6,9,11]$. Despite melanocytoma being a benign tumour, treatment should be performed to prevent intraocular spread, which will ultimately result in loss of the globe due to uveitis, potential retinal detachment or secondary glaucoma [9]. Previous reports of surgical management of canine limbal neoplasia have included full-thickness corneoscleral resection followed by repair with preserved homologous corneoscleral tissue, xenogeneic porcine small intestinal submucosa or synthetic polytetrafluoroethylene, with enucleation required in some cases $[2,5,6,9,11]$. Surgical debulking of limbal melanomas, followed by diode laser photocoagulation has been described in dogs [10]. The choice of treatment is influenced by the tumour size and location, availability of equipment and materials, clinician expertise and possibly the cost of treatment [9]. In the present case, due to the location and size of the mass and the absence of intraocular invasion, surgical removal including a margin of normal tissue was performed. With this surgical procedure, the intention was to preserve the eyeball and maintain vision. In addition to the surgical treatment, analgesic and anti-inflammatory eye drops were prescribed to reduce postoperative complications. A topical antibiotic was used to avoid postoperative contamination. Cyclosporin A $0.2 \%$ eye drops were applied twice daily for 2 months to reduce graft rejection. Complications associated with full-thickness excision and repair of canine limbal neoplasia include anterior uveitis, synechia, dyscoria, hyphema, iris prolapse, conjunctival cyst formation and graft failure $[5,6,9,11]$. In the present case, minimal intraocular inflammation was observed. The postoperative complications included focal corneal oedema, corneal neovascularization, formation of granulation tissue, and minimal corneal pigmentation. These complications have all been reported in previous studies that performed penetrating keratoplasty, and were considered clinically insignificant $[5,6,9,11]$. No other complication reported in the literature was observed during the 3-year postoperative follow-up period. No studies in the literature have compared surgical treatment alone with surgical excision in combination with additional therapies such as cryotherapy. The prognosis is favourable for maintaining both the globe and vision. However, melanocytoma can recur after surgical removal [1]. Deep surgical excision, often combined with a tissue graft, performed early in the course of growth offers the best prognosis for the eye $[5,6,9,11]$. To the best of our knowledge, this is the first report of the use of a fresh homologous corneoscleral graft to repair a surgical defect resulting from excision of a canine limbal neoplasia. The outcome of the present case report is similar to that described previously following penetrating sclerokeratoplasty and frozen homologous corneoscleral grafting for the management of extensive canine epibulbar melanocytomas in dogs [6]. However, in the present report, we did not observe corneal fibrosis, abundant pigmentation or lipid keratopathy. In the current case, the treatment appeared to be successful because our patient is still alive, and presented no evidence of metastasis or recurrence 3 years after treatment. This report demonstrates extremely good efficacy of a fresh homologous corneoscleral graft for maintaining the eye and preserving vision. Surgical excision combined with fresh homologous corneoscleral graft was effective for the treatment of limbal melanocytoma in a dog. Besides, this case report highlights the importance of diagnosis and early treatment for preventing ocular spread, in order to preserve visual function.

\section{MANUFACTURERS}

${ }^{1}$ Allergan. São Paulo, SP, Brazil.
${ }^{2}$ Alcon Laboratórios do Brasil Ltda. São Paulo, SP, Brazil.
${ }^{3}$ Syntec do Brasil Ltda. Santana de Parnaíba, SP, Brazil.
${ }^{4}$ Sanofi Aventis Farmacêutica Ltda. São Paulo, SP, Brazil.
${ }^{5}$ AstraZeneca do Brasil Ltda. Cotia, SP, Brazil.
${ }^{6}$ Instituto BioChimico Indústria Farmacêutica Ltda. Rio de Janeiro,
RJ, Brazil.
${ }^{7}$ Cristália Prod. Quím. Farm. Ltda. São Paulo, SP, Brazil.
${ }^{8}$ Zoetis Indústria de Produtos Veterinários Ltda. Campinas, SP, Brazil.
${ }^{9}$ Novartis Biociências S.A. São Paulo, SP, Brazil.
${ }^{10}$ Farmácia Ophthalmos. São Paulo, SP, Brazil.

Declaration of interest. The authors report no conflicts of interest. The authors alone are responsible for the content and writing of the paper. 


\section{REFERENCES}

1 Donaldson D., Sansom J. \& Adams V. 2006. Canine limbal melanoma: 30 cases (1992-2004). Part 2. Treatment with lamellar resection and adjunctive strontium-90 plesiotherapy - efficacy and morbity. Veterinary Ophthalmology. 9(3): 179-185.

2 Featherstone H.J., Renwick P., Heinrich C.L.\& Manning S. 2009. Efficacy of lamellar resection, cryotherapy, and adjunctive grafting for the treatment of canine limbal melanoma. Veterinary Ophthalmology. 12(Suppl 1): 65-72.

3 Giuliano E.A., Chappel R., Fischer B. \& Dubielzig R.R. 1999. A matched observational study of canine survival with primary intraocular melanocytic neoplasia. Veterinary Ophthalmology. 2(3): 185-190.

4 Hesse K.L., Fredo G., Guimarães L.L.B., Reis M.O., Pigatto J.A.T., Pavarini S.P., Driemeier D. \& Sonne L. 2015. Neoplasmas oculares e de anexos em cães e gatos no Rio Grande do Sul: 265 casos (2009-2014). Pesquisa Veterinária Brasileira. 35(1): 49-54.

5 Lewin G.A. 1999. Repair of a full thickness corneoscleral defect in a German shepherd dog using porcine small intestinal submucosa. The Journal of Small Animal Practice. 40(7): 340-342.

6 Maggio F., Pizzirani S., Peña M.T., Leiva H. \& Pirie C.G. 2013. Surgical treatment of epibulbar melanocytomas by complete excision and homologous corneoscleral grafting in dogs: 11 cases. Veterinary Ophthalmology. 16(1): 56-64.

7 McMullen R.J., Clode A.B., Pandiri A.K., Malarkey D.E., Michau T.M. \& Gilger B.C. 2008. Epibulbar melanoma in a foal. Veterinary Ophthalmology. 11(Suppl 1): 44-50.

8 Naumann G.O.\& Rummelt V.1996. Block excision of tumors of the anterior uvea. Report on 68 consecutive patients. Veterinary Ophthalmology. 103(12): 2017-2027.

9 Norman J.C., Urbanz J.L. \& Calvarese S.T. 2008. Penetrating keratoscleroplasty and bimodal grafting for treatment of limbal melanocytoma in a dog. Veterinary Ophthalmology. 11(5): 340-345.

10 Sullivan T.C., Nasisse M.P., Davidson M.G.\& Glover T.L.1996. Photocoagulation of limbal melanoma in dogs and cats: 15 cases (1989-1993). Journal of the American Veterinary Medical Association. 208(6): 891-894.

11 Wilkie D.A.\& Wolf E.D. 1991. Treatment of epibullbar melanocytoma in adog, using full thickness eyewall resection and synthetic graft. Journal of the American Veterinary Medical Association. 198(6): 1019-1022.

12 Yang S.F., Liang S.L., Yang H.L., Liu C.H. \& Wang P.C. 2017. Case report: excision, amniotic membrane graft, cryotherapy and intralesional mitomycin c injection for the treatment of a limbal melanoma in a dog. Taiwan Veterinary Journal. 43(1): 11-16. 